\title{
FATORES CONTINGENCIAIS E A IMPORTÂNCIA ATRIBUIIDA AOS INDICADORES DE DESEMPENHO: UMA ANÁLISE EM EMPRESAS DE CONSTRUÇÃO CIVIL DA CIDADE DE RECIFE, PE
}

Contingency factors and the importance attached to performance indicators: an analysis in civil construction companies in the city of Recife,

PE

Herivânio Torres Bandeira

E-mail: herivanio@hotmail.com

Mestre em Controladoria pela Universidade Federal Rural de Pernambuco; Especialista em Gestão

Pública pela Universidade Federal Rural de Pernambuco; Técnico Administrativo do Ministério

Público Federal.

https://orcid.org/0000-0002-1726-5625

Aldo Leonardo Cunha Callado

E-mail: aldocallado@yahoo.com.br

Doutor em Agronegócios pela Universidade Federal do Rio Grande do Sul; Mestre em Administração na área de Finanças de Empresas pela Universidade Federal da Paraíba; Professor Associado do Departamento de Finanças e Contabilidade da Universidade Federal da Paraíba. Endereço para contato: Cidade Universitária, 58051-900, João Pessoa, Paraíba, Brasil. https://orcid.org/0000-0002-5393-6094 


\section{Resumo}

Este trabalho teve por objetivo investigar o nível das relações estabelecidas entre fatores contingenciais de empresas pertencentes ao setor de construção civil da Cidade de Recife (PE) e a importância atribuída pelos gestores aos indicadores de desempenho classificados nas perspectivas do Balanced Scorecard (BSC). Nessa perspectiva, foi analisada a importância atribuída aos indicadores de desempenho, sob a ótica da teoria da contingência. Quanto aos procedimentos metodológicos, utilizou-se como instrumento de coleta de dados o questionário, que foi aplicado junto a gestores de 20 empresas do setor da construção civil localizadas na Cidade de Recife. Para análise dos dados coletados foi utilizado o teste não paramétrico coeficiente de correlação de Spearman, com o auxílio do software STATISTICA for Windows. A análise dos resultados evidenciou poucas relações significativas entre as características dos gestores com indicadores de desempenho, sendo a relação da variável faixa etária com o indicador desperdícios a única a alcançar magnitude forte. Também foram evidenciadas algumas relações significativas entre as características das empresas e os indicadores de desempenho, sendo as relações da variável prioridade estratégica com os indicadores (pontualidade de entrega, flexibilidade de volume operacional, entregas no prazo, satisfação dos empregados e gestão de risco) as únicas a apresentarem magnitude forte. As contribuições da pesquisa podem se realizar no campo de estimular outros pesquisadores que pretendam desenvolver estudos correlatos em outras localidades e até mesmo em outros segmentos de mercado, assim como por servirem como parâmetro para reflexão das empresas do setor pesquisado acerca do tema aqui discutido.

Palavras-chave: Indicadores de desempenho. Fatores contingenciais. Construção civil.

\section{Abstract}

This work aims to investigate the level of relations established between contingency factors of Recife's civil construction enterprises, as well as to investigate the importance assigned to performance indicators by its managers in Balanced Scorecard (BSC) perspective. Therefore, the contingency theory was the approach given to the analysis of the importance of performance indicators. As for the methodology, a survey was conducted and applied to 20 managers from civil construction enterprises located in Recife. The data analysis was conducted by the measure of Spearman's correlation coefficient with the support of STATISTICA for Windows software. The analysis of results highlighted few significant relations between managers' characteristics and performance indicators, so that the variables of age range and wastage indicator were the only ones to reach strong magnitude. Moreover, significant relations between enterprises with performance indicators were noticed, so that the relation between strategic priority and indicators (such as delivery punctuality, operational volume flexibility, on-time delivery, employee satisfaction and risk management) were the only ones with significant strength. The research contributions can be addressed to other researchers who intend to develop co-related studies in other locations, even in other market segments, as well as can be attached as a parameter for enterprises involved in the same researched market sector.

Keywords: Performance indicators. Contingency factors. Civil construction.

\section{INTRODUÇÃO}

O aumento da concorrência nos mercados cada vez mais integrados e globalizados tem provocado nas organizações a preocupação por vantagens competitivas (Siqueira, 2014). Dessa forma, a inclusão de novas estratégias organizacionais na gestão tem sido uma 
prática constante nas organizações, com o objetivo de buscar mecanismos que permitam a constante melhoria dos serviços e produtos. Nesse contexto, dinâmico e de complexidade, característico do ambiente empresarial contemporâneo, diversos fatores como globalização e inovações tecnológicas são responsáveis por ocasionar mudanças na maneira pela qual as organizações são geridas (Araújo et al., 2014).

Na gestão estratégica, as informações sobre o desempenho dos ambientes interno e externo norteiam a tomada de decisões, buscando a eficácia empresarial e a melhoria de resultados e de posição competitiva no mercado no qual atua (Callado et al., 2008). Segundo Siqueira (2014), para avaliar o desempenho, as empresas se utilizam de sistemas de medição que possibilitam gerenciar os resultados de suas atividades por meio de indicadores.

Conforme Mainardes et al. (2011), a estratégia nas organizações enquanto campo de estudo é mais recente que sua prática, e o seu conhecimento ainda está em construção, porém a estratégia sempre esteve ligada à antecipação de cenários e aos planos de ação a serem desencadeados neles. Dessa forma, os gestores formulam indicadores que possibilitam monitorar a implementação de estratégias, bem como o acompanhamento do ambiente, visando ajustar reforços e alinhar as estratégias competitivas (Nakamura \& Mineta, 2001).

A teoria da contingência explica como os fatores externos influenciam no crescimento e sobrevivência das organizações. Nesse sentido, Beuren e Fiorentin (2014) asseveram que ambientes externos cada vez mais incertos têm demandado dos gestores informações úteis e tempestivas para acompanhar o desempenho organizacional e obter vantagem competitiva.

Nessa perspectiva, para se tornar competitiva, a organização precisa elaborar estratégias, mas também precisa medir, comparar e tomar decisões por meio de indicadores, ou seja, as estratégias precisam abranger novas perspectivas, financeiras e não financeiras, para melhorar o desempenho e manter a empresa no mercado (Maltempi, 2014).

No escopo desta pesquisa encontra-se a indústria da construção civil, submetida a um cenário no qual as empresas estão sujeitas a modificações contínuas em suas atividades e estratégias, bem como apresentam diversos fatores contingentes, a exemplo da variação das taxas de juros, oscilação do crédito, mudanças no mercado de trabalho, alterações na legislação, entre outros. Conforme asseveram Beuren e Fiorentin (2014), a postura estratégica das organizações está associada a fatores contingentes, como ameaças internas e externas, e é afetada por restrições políticas, econômicas e culturais.

Nesse sentido, Klein e Almeida (2017) afirmam que os fatores contingenciais direcionam a tomada de decisão, protegendo a organização da incerteza, enquanto Silva e Callado (2018a) asseguram que os setores econômicos têm procurado se adaptar às modificações enfrentadas no mercado competitivo, necessitando conhecer o desempenho de suas ações. 
Diante do exposto, o artigo apresenta a seguinte pergunta de pesquisa: existe correlação entre fatores contingenciais e a importância atribuída pelos gestores aos indicadores de desempenho classificados nas perspectivas do Balanced Scorecard (BSC)?

Portanto, o objetivo da pesquisa que deu origem ao artigo consistiu em investigar o nível das relações estabelecidas entre fatores contingenciais de empresas pertencentes ao setor de construção civil da Cidade de Recife, PE, e a importância atribuída pelos gestores aos indicadores de desempenho classificados nas perspectivas do Balanced Scorecard (BSC).

Justifica-se a relevância da pesquisa pelo fato de se buscar suprir uma lacuna identificada, que é a escassez de estudos abordando a temática envolvendo indicadores de desempenho organizacional e fatores contingenciais nas empresas da construção civil da Cidade do Recife, PE. A pesquisa torna-se relevante também por se tratar de um setor de grande importância econômica, com capacidade positiva de distribuição de renda por absorver expressivo contingente de mão de obra, especialmente trabalhadores menos qualificados (Silva et al., 2015), e fomentar setores econômicos associados, como: cimento e concreto, extração mineral, máquinas e equipamentos, produtos siderúrgicos, materiais básicos e serviços especializados para construção (Santos, 2015).

\section{REFERENCIAL CONCEITUAL}

\subsection{FATORES CONTINGENCIAIS}

A Teoria da Contingência foi estruturada com base nas Teorias Neobehaviorista e Sistêmica, as quais consideram a organização como um sistema aberto e cujas variáveis internas são influenciadas pelo meio no qual ela está inserida (Cosso \& Oliveira, 2013; Klein \& Almeida, 2017). O pressuposto fundamental da Teoria da Contingência é a adaptação da organização por meio da interação de fatores contingenciais, isolados ou em conjunto. Nesse contexto, a estrutura mais eficiente é a que se apresenta em conformidade com a estratégia, o porte, a tecnologia e o ambiente onde a organização opera (Espejo, 2008).

Enquanto sistema aberto, a organização interage com o ambiente e com outros fatores contingenciais, como tecnologia, estrutura, estratégia, porte, entre outros (Klein \& Almeida, 2017). Nesse contexto, os fatores contingenciais são fatores caracterizados por variáveis que podem influenciar o processo decisório e afetar a estrutura organizacional (Marques \& Souza, 2010; Santos et al., 2016). Beuren e Fiorentin (2014) defendem que atualmente os fatores contingenciais abordados na literatura são os seguintes: ambiente externo, tecnologia, estrutura, estratégia e porte organizacional. 
Cabe ressaltar que o artigo se limitou a explorar os seguintes fatores contingenciais: características dos gestores (faixa etária, escolaridade e experiência profissional) e características das empresas (tempo de atuação da empresa no mercado, número de empregados, estrutura hierárquica, grau de formalização dos processos organizacionais e prioridade estratégica).

A opção pelos supracitados fatores contingenciais foi motivada pelo fato de já terem sido adotados em trabalhos anteriores (Montenegro \& Callado, 2019; Silva \& Callado, 2018a, 2018b; Silva et al., 2016), os quais investigaram características internas e externas das organizações com a finalidade de compreender o nível de influência dessas características nas decisões organizacionais.

Montenegro e Callado (2019) estudaram os fatores contingenciais associados às características das empresas, dos gestores e das práticas de mensuração de desempenho em empresas do setor de confecções do vestuário. Silva e Callado (2018b) analisaram as relações entre fatores contingenciais e a importância atribuída aos indicadores de desempenho nas empresas de construção civil de João Pessoa, PB. Silva e Calado (2018a) analisaram a aderência de indicadores de desempenho associados às perspectivas do BSC em organizações atuantes na construção civil. Silva et al. (2016) analisaram a significância das relações entre o uso de indicadores de desempenho e fatores contingenciais de empresas da construção civil de João Pessoa, PB.

O ambiente é considerado um fator de contingência externo à organização, ou seja, trata-se do contexto no qual a organização está envolvida. Conforme Leite et al. (2015), - termo ambiente é utilizado para descrever as diversas características do ambiente organizacional que influenciam no sistema de controle gerencial.

Para Jacomossi et al. (2018), a totalidade dos fatores que compõem o ambiente organizacional interno (pessoal, funcionalidade e nível organizacional) e externo (clientes, fornecedores, concorrentes e tecnológico) pode impactar a tomada de decisão, tendo em vista as incertezas atreladas a tais fatores e suas respectivas informações.

Otley (2016) destaca que o aspecto mais amplamente pesquisado do ambiente é a incerteza e que a variável mais comumente utilizada é uma medida da incerteza ambiental percebida. Chenhall (2006), por sua vez, esclarece que a incerteza, por si só, não fornece uma descrição abrangente do ambiente, pois é encontrada uma variedade de variáveis para caracterizar o ambiente externo, como turbulência, hostilidade, diversidade e complexidade.

Outro fator contingencial é a tecnologia, que no ambiente organizacional está relacionada ao conjunto de informações organizadas de diversos tipos, provenientes de várias fontes, obtidas por meio de diversos métodos e que são utilizadas na produção de bens e serviços (Fleury, 1990). 
A tecnologia da informação tem contribuído para aumentar a eficiência da produção e também do produto, além de ser usada como ferramenta de comunicação e gestão empresarial, fazendo com que as empresas se mantenham competitivas nos mercados em que operam (Rossetti \& Morales, 2007). Conforme Hyvonen (2007), "o termo tecnologia da informação refere-se à ênfase de uma empresa em aplicações avançadas de sistemas de informação" (p. 344), e a utilização da tecnologia da informação por parte da organização fornece uma dimensão adicional à relação entre as medidas de desempenho e sua estratégia.

Segundo Chenhall (2003):

\begin{abstract}
A tecnologia tem muitos significados no comportamento organizacional. Em um nível geral, a tecnologia refere-se a como os processos de trabalho da organização operam (a maneira como as tarefas transformam entradas em saídas) e inclui hardware (como máquinas e ferramentas), materiais, pessoas, software e conhecimento (p. 139).
\end{abstract}

O fator contingencial estratégia está relacionado ao estabelecimento de quais caminhos ou cursos de ação devem ser seguidos para o alcance de metas e objetivos organizacionais. Nesse contexto, a estratégia relaciona-se com a maneira de se utilizar adequadamente os recursos visando à maximização das oportunidades (Oliveira, 2005). De acordo com Porter (1999), "estratégia é criar uma posição exclusiva e valiosa envolvendo um diferente conjunto de atividades" (p. 63).

A estratégia, "em certo sentido, não é um elemento de contexto, mas sim o meio pelo qual os gerentes podem influenciar a natureza do ambiente externo, as tecnologias da organização, os arranjos estruturais e a cultura de controle e o Sistema de Controle Gerencial" (Chenhall, 2006, p. 184). Segundo o mesmo autor, as pesquisas reconhecem que os gerentes têm uma "escolha estratégica" por meio da qual podem posicionar suas organizações em ambientes específicos.

O porte organizacional é outro fator contingencial, o qual pode ser mensurado utilizando-se critérios quantitativos e/ou qualitativos. Os critérios quantitativos são critérios econômicos, como lucros, valor do capital, ativos, volume de vendas, valorização de ações e número de empregados, os quais dão uma dimensão do porte da organização (Chenhall, 2006; Leone, 1991). Os critérios qualitativos são largamente utilizados para definir o porte de uma organização e são sincronizados com os estilos de gestão, perfil e atitudes dos gestores e suas percepções do ambiente externo (Leone, 1991).

Conforme Abdel-Kader e Luther (2008), o porte de uma organização é um fator importante que afeta a estrutura e outros arranjos de controle. À medida que as organizações se tornam maiores, aumenta a necessidade de lidar com grandes quantidades de informações, o que acarreta a instituição de controles, como regras, documentação, especialização de 
papéis e funções, hierarquias ampliadas e maior descentralização de estruturas hierárquicas (Chenhall, 2006).

Por fim, tem-se o fator contingencial estrutura organizacional, que pode ser entendido como a maneira pela qual a organização divide seu trabalho em tarefas distintas e depois consegue a coordenação entre elas. As variáveis utilizadas para descrever as estruturas organizacionais podem ser agrupadas em duas dimensões principais: complexidade estrutural e controle estrutural (Maguire, 2003).

A complexidade estrutural refere-se ao grau de diferenciação (vertical, funcional, espacial) dentro da organização, incluindo o grau de especialização ou divisão do trabalho e o número de níveis hierárquicos. Já o controle estrutural refere-se aos aparatos administrativos (administração, formalização, centralização) formais que a organização institui para conseguir coordenação e controle entre seus trabalhadores e o trabalho (Maguire, 2003).

A estrutura organizacional é um fator contingencial interno, controlável pela organização e que, conforme Chenhall (2006), está relacionado à especificação formal de diferentes papéis a serem exercidos pelos membros de uma organização ou tarefas para os grupos, para garantir que as atividades da organização sejam executadas. Para Donaldson (2015), a estrutura organizacional refere-se às características como grau de especialização dos papéis em uma organização e centralização da tomada de decisões.

\subsection{INDICADORES DE DESEMPENHO}

As medidas de desempenho auxiliam a organização a estabelecer o grau de evolução em que seus processos se encontram e fornecem informações adequadas para que sejam tomadas ações preventivas ou corretivas em busca das metas e objetivos estabelecidos (Heinzen et al., 2013).

Segundo Moreira (2002), "o indicador é o resultado de uma medida ou de mais medidas que tornam possível a compreensão da evolução do que se pretende avaliar a partir dos limites (referências ou metas) estabelecidos" (p. 15). Desse modo, Wernke e Junges (2017) asseveram que "um indicador não é apenas uma estatística, pois representa uma variável que assume um valor em um tempo específico" (p. 59).

Os indicadores de desempenho possuem distintas classificações, tais como: financeiros e não financeiros; internos e externos; de tendência e de ocorrência; quantitativos e qualitativos (Marquezan et al., 2013). Ainda conforme os mesmos autores, dentre essas classificações, a mais comumente utilizada é a que diferencia os indicadores de desempenho entre financeiros e não financeiros.

Indicadores financeiros "são os indicadores mais tradicionalmente utilizados pelas empresas para a avaliação de seu desempenho", esses indicadores "objetivam orientar a 
organização para a tomada de decisão com relação a questões econômicas e patrimoniais" (Marquezan et al., 2013, p. 48). Os indicadores financeiros expressam em unidades monetárias os aspectos mensuráveis da organização e estão relacionados com as "abordagens que utilizam medidas puramente financeiras tais como lucratividade, custo de mão de obra, retorno sobre investimento, lote econômico e fluxo de caixa" (Tezza et al., 2010, p. 78).

Os indicadores não financeiros apresentam a capacidade de transmitir informações com maior facilidade e podem ser expressos em unidades de medida de mais fácil interpretação, tais como quantidades, metros, volumes e horas. "Os indicadores não financeiros podem ser considerados estratégicos para a gestão dos negócios e para a formação de vantagens competitivas" (Wernke \& Junges, 2017, p. 57). Nesse contexto, os indicadores não financeiros servem como molas propulsoras para motivar o desempenho dos processos em toda a organização, além de possibilitarem correções durante o curso das operações em virtude de transmitirem informações de tendências (Marquezan et al., 2013).

\section{PROCEDIMENTOS METODOLÓGICOS}

\subsection{TIPOLOGIA DA PESQUISA}

Com relação aos objetivos, esta pesquisa pode ser classificada como exploratória e descritiva acerca do tema mensuração de desempenho, visto que a pesquisa descritiva observa, registra, analisa e correlaciona fatos ou fenômenos (variáveis) sem manipulálos (Cervo et al., 2007). No tocante à abordagem do problema de pesquisa, esta é uma investigação predominantemente quantitativa, visto que nas pesquisas quantitativas tudo pode ser quantificável, o que significa traduzir, em números, opiniões e informações para classificá-las e analisá-las, fazendo uso de técnicas estatísticas (Prodanov \& Freitas, 2013).

Como procedimento metodológico, optou-se por uma survey, ou pesquisa de levantamento, a qual, segundo Prodanov e Freitas (2013), envolve a interrogação direta das pessoas cujo comportamento se quer conhecer, utilizando-se de questionários. $\mathrm{O}$ instrumento do qual se fez uso para a coleta dos dados analisados nesta pesquisa foi um questionário com perguntas fechadas. Para tanto, foi utilizada uma adaptação do questionário contido no protocolo de pesquisa desenvolvido e aplicado por Silva e Callado (2018b), que investigaram fatores contingenciais e indicadores de desempenho na indústria da construção civil da Cidade de João Pessoa, PB. O questionário foi aplicado com os gestores e/ou proprietários das empresas pesquisadas com experiência prática acerca do tema abordado. $O$ contato com os gestores investigados se realizou através de ligações telefônicas, envio de e-mails e 
contato presencial. A coleta de dados teve início em outubro de 2018 e se estendeu até o início de fevereiro de 2019.

Para dimensionar o universo da pesquisa foi realizada consulta ao sítio institucional do Sindicado da Indústria da Construção Civil no Estado de Pernambuco (Sinduscon-PE) para verificar a relação das empresas associadas àquele sindicato. Verificou-se que a listagem de empresas associadas era composta por 162 organizações, das quais 123 estavam sediadas na Cidade de Recife, dentre as quais apenas 94 exerciam atividade econômica principal compatível com o objetivo da pesquisa e, portanto, compuseram o seu universo.

A amostra de pesquisa é não probabilística, escolhida por acessibilidade, constituída por 20 empresas que aceitaram participar da pesquisa. Dessa forma, a amostra representa, aproximadamente, $21,28 \%$ da população.

\subsection{VARIÁVEIS INVESTIGADAS}

Para operacionalização desta pesquisa foram utilizados três grupos de variáveis. $O$ primeiro grupo foi composto por fatores internos relacionados às características dos gestores das organizações pesquisadas. No segundo grupo foram reunidos os fatores contingentes relativos às características das empresas participantes da pesquisa.

No tocante ao terceiro grupo, foram elencadas variáveis para investigar a importância atribuída aos indicadores de desempenho associáveis às perspectivas do Balanced Scorecard, em que foram considerados 36 indicadores distribuídos dentre as dimensões do BSC. Os grupos de variáveis podem ser visualizados na Tabela 1.

Tabela 1

Relação de variáveis distribuídas nas dimensões do BSC

\begin{tabular}{|c|c|c|}
\hline \multirow{2}{*}{$\begin{array}{c}\text { Perspectivas } \\
\text { do BSC }\end{array}$} & \multicolumn{2}{|l|}{ Variáveis } \\
\hline & Indicadores de desempenho (Grupo 3) & Grupos 1 e 2 \\
\hline Financeira & $\begin{array}{l}\text { Lucratividade; Rentabilidade; Faturamento; Crescimento das } \\
\text { vendas; Custos totais; Custos unitários; Fluxo de caixa; Retorno do } \\
\text { investimento; Inventários; Preço dos produtos; e Capital de giro. }\end{array}$ & Grupo 1 (Faixa Etária \\
\hline Clientes & $\begin{array}{l}\text { Quantidade de clientes; Novos clientes; Satisfação de clientes; } \\
\text { Qualidade dos produtos; Devolução de produtos; Tempo de entre- } \\
\text { ga; Pontualidade de entrega; Responsividade; e Crescimento da } \\
\text { participação de mercado. }\end{array}$ & $\begin{array}{l}\text { de Escolaridade; Experi- } \\
\text { ência Profissional). } \\
\text { Grupo } 2 \text { (Tempo de } \\
\text { atuação da empresa no }\end{array}$ \\
\hline $\begin{array}{l}\text { Processos } \\
\text { Internos }\end{array}$ & $\begin{array}{l}\text { Tempo de realização das operações; Flexibilidade de entrega; } \\
\text { Tempo de estocagem; Fornecedores; Flexibilidade de volume ope- } \\
\text { racional; Uso da capacidade instalada; Pós-venda; Entregas no } \\
\text { prazo; e Desperdícios. }\end{array}$ & $\begin{array}{l}\text { mercado; Número de } \\
\text { empregados; Estrutura } \\
\text { hierárquica; Grau de } \\
\text { formalização dos pro- }\end{array}$ \\
\hline $\begin{array}{l}\text { Aprendizagem } \\
\text { e Crescimento }\end{array}$ & $\begin{array}{l}\text { Produtividade dos empregados; Satisfação dos empregados; Rota- } \\
\text { tividade de empregados; Flexibilidade dos empregados; Inovação } \\
\text { nos produtos; Investimentos em treinamento; e Gestão de risco. }\end{array}$ & $\begin{array}{l}\text { cessos operacionais; e } \\
\text { Prioridade estratégica). }\end{array}$ \\
\hline
\end{tabular}


Nesta pesquisa, a aferição do grau de importância atribuída aos indicadores associáveis às perspectivas do BSC foi operacionalizada por meio de Escala do tipo Likert, em que as variáveis foram representadas matematicamente por 01 para o grau mínimo de importância e por 04 para o grau máximo.

\subsection{TÉCNICAS DE ANÁLISE ESTATÍSTICA}

Para atingir o objetivo proposto, a análise dos dados foi submetida ao teste não paramétrico coeficiente de correlação de Spearman. O teste foi aplicado com o objetivo de analisar as relações entre os fatores contingenciais investigados e a importância atribuída aos indicadores de desempenho organizacional. Os procedimentos foram realizados com a utilização do software STATISTICA for Windows. Na aplicação do coeficiente de correlação de Spearman ( $r$ ) foi considerado o nível de significância de $95 \%(=0,05)$ como referência mínima aceitável para determinar a significância estatística. Os critérios utilizados para interpretar a magnitude da correlação foram os de Franzblau (1958). De acordo com Franzblau (1958 apud Pereira et al., 2016): se $r=0$, não existe relação linear entre as variáveis; se $|r|<0,20$, a correlação é negligenciável; se $0,20<|r|<0,40$, a correlação é fraca; se 0,40 < |r|<0,60, a correlação é moderada; se $0,60<|r|<0,80$, a correlação é forte; e se $|r|>0,80$, a correlação é muito forte.

\section{RESULTADOS DA PESQUISA}

\subsection{RELAÇÕES ENTRE A IMPORTÂNCIA ATRIBUÍDA AOS INDICADORES DE DESEMPENHO E CARACTERÍSTICAS DOS GESTORES}

Inicialmente foram consideradas as relações entre a importância atribuída aos indicadores de desempenho da perspectiva financeira e as características dos gestores. Os resultados estão apresentados na Tabela 2. 
Tabela 2

Relações entre indicadores de desempenho da perspectiva financeira e características dos gestores

\begin{tabular}{lccr}
\hline \multicolumn{1}{c}{ Indicadores } & \multicolumn{2}{c}{ Correlação } & V3 \\
\cline { 2 - 4 } & V1 & V2 & 0,086851 \\
\hline Lucratividade & 0,078602 & 0,244352 & $-0,073659$ \\
Rentabilidade & 0,075822 & 0,096757 & $-0,055693$ \\
Faturamento & 0,035939 & 0,162932 & $-0,275641$ \\
Crescimento das vendas & $-0,359805$ & 0,294792 & 0,128489 \\
Custos totais & 0,276974 & $-0,149696$ & 0,224045 \\
Custos unitários & 0,313489 & $-0,051434$ & $-0,138682$ \\
Fluxo de caixa & $-0,193663$ & 0,282440 & $-0,005717$ \\
Retorno do investimento & 0,000000 & $-0,062994$ & $-0,311199$ \\
Inventários & $-0,315995$ & 0,159965 & $-0,383254$ \\
Preço dos produtos & $-0,266464$ & $-0,061207$ & 0,004587 \\
Capital de Giro & 0,044009 & 0,212284 & \\
\hline
\end{tabular}

Nota. V1 - Faixa Etária; V2 - Escolaridade; e V3 - Experiência Profissional.

Analisando os resultados constantes na Tabela 2 é possível observar que as relações estabelecidas entre as variáveis analisadas, após a aplicação do coeficiente de correlação de Spearman, resultaram enquadradas no nível de magnitude fraca e negligenciável. Destaquese que a variável V1 (faixa etária) apresentou correlação $\mathrm{O}$ (zero) com o indicador retorno do investimento, o que sugere independência da importância atribuída a esse indicador em relação a essa variável.

Tinoco et al. (2011) asseveram que, numa visão pragmática, as organizações têm como princípio de gestão dar ênfase aos elementos que afetam sua sobrevivência. Dessa forma, os indicadores financeiros recebem atenção dos gestores por serem premissa para continuidade das organizações, fatos não observados nas correlações aqui analisadas. Logo, esses resultados sugerem que as características dos gestores da amostra de empresas pesquisadas pouco influenciam a importância atribuída aos indicadores de desempenho da perspectiva financeira do BSC.

Os resultados aqui evidenciados vão de encontro aos resultados obtidos por Silva e Callado (2018b), visto que no trabalho desses autores ficou demonstrado que uma característica dos gestores, a escolaridade, repercute na importância que estes atribuem aos indicadores associados à dimensão financeira do BSC.

Na sequência foram analisadas as possíveis correlações entre a importância atribuída aos indicadores de desempenho da perspectiva clientes e as características dos gestores das empresas pesquisadas, cujos resultados estão dispostos na Tabela 3. 
Tabela 3

Relações entre indicadores de desempenho da perspectiva clientes e características dos gestores

\begin{tabular}{lccc}
\hline \multirow{2}{*}{ Indicadores } & \multicolumn{3}{c}{ Correlação } \\
\cline { 2 - 4 } & V1 & V2 & V3 \\
\hline Quantidade de clientes & $-0,354192$ & 0,156457 & $-0,370042$ \\
Novos clientes & $-0,271090$ & 0,307871 & $-0,108601$ \\
Satisfação de clientes & 0,066461 & $\mathbf{0 , 4 9 4 8 3 0}$ & $-0,155735$ \\
Qualidade dos produtos & 0,207748 & 0,169620 & 0,022063 \\
Devolução de produtos & 0,041934 & 0,070975 & 0,201507 \\
Tempo de entrega & 0,049112 & 0,063258 & $-0,082282$ \\
Pontualidade de entrega & $-0,061079$ & 0,134687 & $-0,124264$ \\
Responsividade & $-0,209363$ & 0,280528 & $-0,190931$ \\
Crescimento da participação de mercado & 0,037907 & $-0,039578$ & 0,118524 \\
\hline
\end{tabular}

Nota. V1 - Faixa Etária; V2 - Escolaridade; e V3 - Experiência Profissional.

Analisando os resultados contidos na Tabela 3 é possível observar que a variável V2 (escolaridade) se correlaciona de forma direta e moderada com o indicador satisfação de clientes. Esse resultado sugere que um maior nível de escolaridade dos gestores da amostra de empresas pesquisadas influencia diretamente a importância atribuída ao indicador relacionado à satisfação de clientes.

Resultados divergentes com os aqui evidenciados foram encontrados por Silva e Callado (2018b), visto que no trabalho desses autores não foram visualizadas correlações estatisticamente significativas entre as características dos gestores e a importância atribuída por eles aos indicadores de desempenho da dimensão clientes do BSC.

$\mathrm{Na}$ sequência foram investigadas as possíveis relações entre a importância dada aos indicadores de desempenho da perspectiva processos internos e as características dos gestores das empresas estudadas, cujos resultados estão apresentados na Tabela 4.

Tabela 4

Relações entre indicadores de desempenho da perspectiva processos internos e características dos gestores

\begin{tabular}{lcrr}
\hline \multirow{2}{*}{ Indicadores } & \multicolumn{3}{c}{ Correlação } \\
\cline { 2 - 4 } & V1 & V2 & V3 \\
\hline Tempo de realização das operações & $-0,151852$ & $\mathbf{0 , 4 1 0 9 4 6}$ & $-0,402489$ \\
Flexibilidade de entrega & $-0,228817$ & 0,020940 & $-0,276489$ \\
Tempo de estocagem & $-0,182098$ & 0,010152 & $-0,368527$ \\
Fornecedores & 0,271930 & 0,053754 & 0,313174 \\
Flexibilidade de volume operacional & $-0,265690$ & 0,039758 & $-0,288189$ \\
Uso da capacidade instalada & $-0,209005$ & $-0,019920$ & $-0,190718$ \\
Pós-venda & $-0,033845$ & $-0,155483$ & $-0,156149$ \\
Entregas no prazo & $-0,249807$ & 0,152145 & $-0,199215$ \\
Desperdícios & $\mathbf{0 , 4 1 0 6 0 0}$ & $-0,383311$ & $\mathbf{0 , 6 4 6 7 2 4}$ \\
\hline
\end{tabular}

Nota. V1 - Faixa Etária; V2 - Escolaridade; e V3 - Experiência Profissional. 
A análise dos dados da Tabela 4 revela que a variável V1 (faixa etária) apresentou correlação direta, de magnitude moderada, com o indicador desperdícios. Esse resultado sinaliza que a faixa etária impacta a importância atribuída ao indicador desperdícios da dimensão processos internos do BSC de tal modo que quanto maior a faixa etária dos gestores da amostra pesquisada, mais importância é dada a esse indicador.

O coeficiente de Spearman também revelou correlação positiva e moderada entre a variável V2 (escolaridade) e o indicador tempo de realização das operações. Esse resultado sugere que o aumento no nível do ensino formal dos gestores das empresas pesquisadas influencia positivamente a importância atribuída ao indicador tempo de realização das operações.

Para a variável V3 (experiência profissional), o coeficiente de Spearman mostrou correlação negativa e moderada com o indicador tempo de realização das operações. Essa variável também apresentou associação positiva e forte com o indicador de desempenho desperdícios. Nesse caso, os resultados da correlação de Spearman sugerem que a experiência profissional do gestor influencia positivamente a importância atribuída ao indicador desperdícios e impacta negativamente a importância atribuída ao indicador tempo de realização das operações.

O cotejamento desses resultados com os valores encontrados por Silva e Callado (2018b) possibilita asseverar que os resultados se complementam, visto que no trabalho dos autores supracitados ficou demonstrado que uma das características dos gestores, a escolaridade, impacta na importância que estes atribuem aos indicadores associados à dimensão processos internos do BSC.

Por último, foram investigadas as possíveis relações entre a importância atribuída aos indicadores de desempenho da perspectiva aprendizagem e crescimento e as características pessoais dos gestores, cujos resultados se encontram consolidados na Tabela 5 .

Tabela 5

Relações entre indicadores de desempenho da perspectiva aprendizagem e crescimento e características dos gestores

\begin{tabular}{lcrr}
\hline \multirow{2}{*}{ Indicadores } & \multicolumn{3}{c}{ Correlação } \\
\cline { 2 - 4 } & V1 & V2 & V3 \\
\hline Produtividade dos empregados & 0,065731 & $-0,130345$ & 0,143915 \\
Satisfação dos empregados & $-0,076187$ & $-0,183140$ & $-0,062786$ \\
Rotatividade de empregados & $-0,174979$ & 0,160369 & $-0,271055$ \\
Flexibilidade dos empregados & $-0,298378$ & $-0,050544$ & $-0,356851$ \\
Inovação nos produtos & $-0,272881$ & $-0,029336$ & $-0,231165$ \\
Investimentos em treinamento & $-0,309219$ & 0,000000 & $-0,035659$ \\
Gestão de risco & $-0,117077$ & $-0,139443$ & $-0,151851$ \\
\hline
\end{tabular}

Nota. V1 - Faixa Etária; V2 - Escolaridade; e V3 - Experiência Profissional. 
Analisando os dados contidos na Tabela 5 é possível observar que as relações estabelecidas entre as variáveis analisadas, após a aplicação do coeficiente de correlação de Spearman, resultaram enquadradas no nível de magnitude entre negligenciável e fraca, conforme critério adotado para análise da magnitude das correlações.

Destaque-se que a variável V2 (escolaridade) apresentou correlação O (zero) com o indicador investimentos em treinamento, o que sugere independência da importância atribuída a esse indicador em relação à variável.

Os resultados contidos na Tabela 5 sugerem que a importância atribuída pelos gestores das empresas pesquisadas aos indicadores de desempenho associáveis à perspectiva aprendizagem e crescimento do BSC é pouco influenciada pelas características dos gestores.

Esses resultados se apresentam diferentes dos resultados encontrados no trabalho de Silva e Callado (2018b), no qual a variável faixa etária apresentou correlação estatisticamente significativa com o indicador de desempenho produtividade dos empregados.

Ao final da análise das correlações envolvendo as características dos gestores e os indicadores de desempenho classificados nas perspectivas do BSC, restou evidenciado que apenas cinco correlações se mostraram estatisticamente significativas, conforme pode ser observado na Tabela 6.

Tabela 6

Resumo das constatações contidas nas correlações estatisticamente significativas

\begin{tabular}{|c|c|c|c|}
\hline \multirow{2}{*}{ Perspectivas do BSC } & \multicolumn{3}{|c|}{ Características dos Gestores x Indicadores de Desempenho } \\
\hline & $\mathrm{V} 1$ & V2 & V3 \\
\hline Financeira & - & - & - \\
\hline Clientes & - & Satisfação de clientes & - \\
\hline Processos internos & $\begin{array}{l}\text { Desper- } \\
\text { dícios }\end{array}$ & Tempo de realização das operações & $\begin{array}{l}\text { Desperdícios } \\
\text { (Tempo de realização das operações) }\end{array}$ \\
\hline $\begin{array}{l}\text { Aprendizagem e } \\
\text { crescimento }\end{array}$ & - & - & - \\
\hline
\end{tabular}

Na Tabela 6 é possível observar que indicadores de apenas duas dimensões do BSC se apresentaram correlacionados com as variáveis (fatores internos) características do perfil dos gestores. A correlação entre a variável experiência profissional e o indicador desperdícios foi a única que atingiu magnitude forte, sugerindo influência da variável na importância atribuída ao indicador.

Esse resultado aqui encontrado pode ser explicado pelo resultado alcançado no trabalho de Sousa et al. (2018), quando esses pesquisadores constataram que todas as construtoras por eles estudadas estavam envolvidas com a filosofia de gestão chamada 
Construção enxuta, que aplica os princípios do Sistema Toyota de Produção na construção civil, o que sugere que este pode ser um movimento da indústria da construção civil no caminho da eliminação do desperdício.

\subsection{RELAÇÕES ENTRE A IMPORTÂNCIA ATIBUÍDA AOS INDICADORES DE DESEMPENHO E AS CARACTERÍSTICAS DAS EMPRESAS}

Inicialmente foram analisadas as relações entre a importância atribuída aos indicadores de desempenho da perspectiva financeira e as características das empresas. As características das empresas dizem respeito ao seu ambiente organizacional interno $e$ externo. Os resultados são apresentados na Tabela 7.

Tabela 7

Relações entre os indicadores de desempenho da perspectiva financeira e as características das empresas

\begin{tabular}{lccccc}
\hline \multicolumn{1}{c}{ Indicadores } & \multicolumn{5}{c}{ Correlação } \\
\cline { 2 - 6 } \multicolumn{1}{c}{ V4 } & \multicolumn{1}{c}{ V5 } & V6 & V7 & V8 \\
\hline Lucratividade & $-0,212576$ & 0,342297 & 0,186348 & $-0,190344$ & 0,312612 \\
Rentabilidade & $-0,237670$ & 0,130867 & 0,072099 & 0,205080 & 0,396117 \\
Faturamento & $-0,180098$ & 0,080053 & $-0,148537$ & $-0,102716$ & 0,090049 \\
Crescimento das vendas & $-0,087282$ & $-0,122927$ & $-0,145075$ & $\mathbf{0 , 4 0 0 8 1 2}$ & 0,358824 \\
Custos totais & $-0,252798$ & 0,234491 & $\mathbf{0 , 4 1 6 9 9 3}$ & $-0,112880$ & 0,367707 \\
Custos unitários & $-0,094756$ & 0,114998 & 0,280263 & $-0,125789$ & 0,142134 \\
Fluxo de caixa & $-0,167248$ & 0,262700 & 0,253152 & $-0,177618$ & 0,371663 \\
Retorno do investimento & 0,000000 & 0,219089 & 0,056108 & 0,246494 & 0,580259 \\
Inventários & $-0,331535$ & 0,193314 & 0,244101 & 0,023636 & $\mathbf{0 , 5 1 5 7 2 1}$ \\
Preço dos produtos & $-0,300690$ & $-0,155431$ & $-0,021379$ & 0,177962 & $\mathbf{0 , 4 6 9 8 2 9}$ \\
Capital de giro & $-0,055869$ & $-0,064874$ & $-0,365448$ & $-0,041203$ & 0,083803 \\
\hline
\end{tabular}

Nota. V4 - Tempo de Atuação da Empresa; V5 - Número de Empregados; V6 - Estrutura Hierárquica; V7 Grau de Formalização de Processos Operacionais; e V8 - Prioridade Estratégica.

Nos resultados apresentados na Tabela 7 é possível observar que as correlações estabelecidas entre as variáveis V4 (tempo de atuação da empresa) e V5 (número de empregados) e os indicadores da perspectiva financeira do BSC, após a aplicação do coeficiente de correlação de Spearman, resultaram enquadradas no nível de magnitude entre negligenciável e fraca, conforme critério adotado para análise da magnitude das correlações. Destaque-se que a variável V4 apresentou correlação 0 (zero) com o indicador retorno do investimento, o que sugere independência da importância atribuída a esse indicador em relação à variável. Esses resultados sugerem que o tempo de atuação da empresa no mercado e o número de empregados são fatores que pouco influenciam na 
importância atribuída aos indicadores de desempenho da perspectiva financeira do BSC na amostra de empresas pesquisadas.

Conforme os resultados da Tabela 7, a variável V6 (estrutura hierárquica) se mostrou relacionada de forma positiva e moderada com o indicador custos totais. Esse resultado sugere que os gestores das empresas pesquisadas mais hierarquizadas, ou seja, empresas mais descentralizadas tendem a atribuir maior importância ao indicador custos totais.

Os resultados da Tabela 7 demonstram também que a variável V7 (grau de formalização de processos operacionais) aparece correlacionada de forma positiva e moderada com o indicador crescimento das vendas. Esse resultado sugere que os gestores da amostra de empresas pesquisadas associam maior crescimento das vendas a um maior nível de formalização dos processos operacionais.

Além disso, os resultados da Tabela 7 demonstram que a variável V8 (prioridade estratégica) apareceu correlacionada de forma positiva e moderada com os indicadores retorno do investimento, inventários e preço dos produtos. Esses resultados sugerem que a prioridade estratégica adotada pelas empresas da amostra pesquisada encoraja os gestores a atribuírem maior importância aos indicadores de desempenho que estão diretamente relacionados ao resultado financeiro.

Os resultados deste estudo divergem, nesse ponto, dos resultados alcançados por Silva e Callado (2018b), pelo fato de na pesquisa desses autores não terem sido visualizadas correlações estatisticamente significativas entre o tempo de atuação, o número de empregados, a estrutura hierárquica e a formalização dos processos operacionais e a importância que os gestores atribuem aos indicadores financeiros da organização.

Em seguida foram analisadas as relações entre a importância atribuída aos indicadores de desempenho da perspectiva clientes e as características das empresas, cujos resultados são apresentados na Tabela 8.

Tabela 8

Relações entre os indicadores de desempenho da perspectiva clientes e as características das empresas

\begin{tabular}{lrrrrr}
\hline \multirow{2}{*}{\multicolumn{1}{c}{ Indicadores }} & \multicolumn{4}{c}{ Correlação } \\
\cline { 2 - 6 } & \multicolumn{1}{c}{ V4 } & \multicolumn{1}{c}{ V5 } & \multicolumn{1}{c}{ V6 } & \multicolumn{1}{c}{ V7 } & V8 \\
\hline Quantidade de clientes & $-0,243199$ & $-0,367623$ & $-0,243872$ & 0,192114 & 0,117096 \\
Novos clientes & $-0,082332$ & $-0,099098$ & 0,003122 & 0,050195 & 0,192109 \\
Satisfação de clientes & 0,029094 & 0,197033 & $-0,099291$ & 0,202552 & 0,310335 \\
Qualidade dos produtos & $-0,062497$ & 0,036051 & $-0,082945$ & 0,113385 & $\mathbf{0 , 4 0 6 2 3 0}$ \\
Devolução de produtos & $-0,373584$ & $-0,492012$ & $-\mathbf{0 , 4 3 6 6 7 3}$ & 0,023144 & 0,130754 \\
Tempo de entrega & $-0,126250$ & $-0,080320$ & $-0,259070$ & 0,273312 & 0,466153 \\
Pontualidade de entrega & $-0,279145$ & 0,115249 & $-0,082329$ & 0,356841 & 0,609984 \\
Responsividade & $-0,369148$ & $-0,468747$ & $-0,399993$ & 0,103726 & 0,184574 \\
Crescimento da participação de mercado & $-\mathbf{0 , 4 3 7 4 7 9}$ & $-0,103237$ & $-0,072577$ & 0,077434 & $\mathbf{0 , 5 1 0 3 9 2}$ \\
\hline
\end{tabular}

Nota. V4 - Tempo de Atuação da Empresa; V5 - Número de Empregados; V6 - Estrutura Hierárquica; V7 Grau de Formalização de Processos Operacionais; e V8 - Prioridade Estratégica. 
Analisando os resultados da Tabela 8, observa-se que a variável V4 (tempo de atuação da empresa) apresentou correlação negativa e moderada com o indicador crescimento da participação de mercado. A variável V5 (número de empregados) apresentou correlação negativa e moderada com os indicadores devolução de produtos e responsividade.

A variável V6 (estrutura hierárquica) apresentou correlação negativa e moderada com o indicador devolução de produtos. Os resultados encontrados sugerem que, na amostra estudada, quanto maior o tempo de atuação das empresas no mercado, quanto maior o número de empregados e quanto mais hierarquizadas são, menos os gestores atribuem importância aos indicadores relacionados com a monitoração do relacionamento da empresa com seus clientes.

A variável V7 (grau de formalização de processos operacionais) só apresentou associações enquadradas no nível de magnitude entre negligenciável e fraca, conforme critério adotado para análise da magnitude das correlações. Esse resultado sugere haver pouca influência dessa variável na importância que os gestores dispensam ao indicador de desempenho da perspectiva clientes do BSC.

A variável V8 (prioridade estratégica) apresentou correlação positiva e moderada com os indicadores qualidade dos produtos, tempo de entrega e crescimento da participação de mercado. Essa variável também apresentou associação positiva e forte com o indicador pontualidade de entrega. Esses resultados sugerem que, na amostra estudada, a prioridade estratégica adotada pelas empresas influencia os gestores a atribuírem maior importância aos indicadores relacionados com a monitoração do relacionamento da empresa com seus clientes.

O comparativo dos resultados alcançados nesse ponto da pesquisa com os valores encontrados por Silva e Callado (2018b) possibilita afirmar que esses resultados se complementam, visto que no trabalho desses autores ficou evidenciado que algumas das características das empresas influenciam a importância atribuída aos indicadores de desempenho associados à perspectiva clientes do BSC.

Na sequência foram pesquisadas as relações entre a importância atribuída aos indicadores de desempenho da perspectiva processos internos e as características das empresas, cujos resultados são apresentados na Tabela 9.

Os resultados evidenciaram que as variáveis V4 (tempo de atuação da empresa), V5 (número de empregados), V6 (estrutura hierárquica) e V7 (grau de formalização de processos operacionais), após a aplicação do coeficiente de correlação de Spearman, apresentaram relações que resultaram enquadradas no nível de magnitude entre negligenciável e fraca, conforme critério adotado para análise da magnitude das correlações. Esses resultados sugerem haver pouca influência dessas variáveis na importância que os gestores dispensam aos indicadores de desempenho da perspectiva processos internos do BSC. Destaque-se que 
V4 apresentou correlação 0 (zero) com o indicador tempo de realização das operações, o que sugere independência da importância atribuída a esse indicador em relação à variável.

Tabela 9

Relações entre os indicadores de desempenho da perspectiva processos internos e as características das empresas

\begin{tabular}{lccccc}
\hline & \multicolumn{4}{c}{ Correlação } \\
\cline { 2 - 6 } \multicolumn{1}{c}{ Indicadores } & V4 & V5 & V6 & V7 & V8 \\
\hline Tempo de realização das operações & 0,000000 & 0,317886 & 0,015754 & 0,226333 & 0,443164 \\
Flexibilidade de entrega & $-0,125375$ & $-0,201143$ & $-0,207356$ & 0,355063 & 0,260394 \\
Tempo de estocagem & $-0,261848$ & $-0,127786$ & $-0,117023$ & 0,343466 & 0,327310 \\
Fornecedores & $-0,009903$ & 0,026708 & $-0,174615$ & 0,079753 & 0,207962 \\
Flexibilidade de volume operacional & $-0,393693$ & 0,104119 & 0,017185 & $-0,039704$ & $\mathbf{0 , 6 3 1 7 3 9}$ \\
Uso da capacidade instalada & $-0,220193$ & 0,230115 & 0,235878 & 0,131538 & $\mathbf{0 , 4 5 8 7 3 5}$ \\
Pós-venda & $-0,028644$ & $-0,035192$ & 0,223752 & 0,067600 & 0,295988 \\
Entregas no prazo & $-0,360375$ & $-0,018898$ & $-0,091102$ & 0,085049 & 0,700729 \\
Desperdícios & $-0,288039$ & $-0,040929$ & $-0,238883$ & $-0,092098$ & 0,018583
\end{tabular}

Nota. V4 - Tempo de Atuação da Empresa; V5 - Número de Empregados; V6 - Estrutura Hierárquica; V7 -

Grau de Formalização de Processos Operacionais; e V8 - Prioridade Estratégica.

A variável V8 (prioridade estratégica) apresentou correlação positiva e moderada com os indicadores tempo de realização das operações e uso da capacidade instalada, além de correlação positiva e forte com os indicadores flexibilidade de volume operacional e entregas no prazo. Esses resultados sugerem que, na amostra de empresas estudadas, a prioridade estratégica adotada influencia a importância atribuída pelos gestores aos indicadores que monitoram a atividade-fim da organização.

O estudo de Silva e Callado (2018b), assim como neste tópico do artigo, também evidenciou que algumas das características das empresas influenciam a importância atribuída pelos gestores aos indicadores de desempenho associados à perspectiva processos internos do BSC. Dessa maneira, pode-se afirmar que esses estudos se complementam, visto que os resultados encontrados convergem na direção de haver correlações significativas entre as variáveis e os indicadores analisados.

Em seguida foram analisadas as relações entre a importância atribuída aos indicadores de desempenho da perspectiva aprendizagem e crescimento e as características das empresas, cujos resultados são apresentados na Tabela 10. 
Tabela 10

Relações entre os indicadores de desempenho da perspectiva aprendizagem e crescimento e as características das empresas

\begin{tabular}{lccccc}
\hline \multicolumn{1}{c}{ Indicadores } & \multicolumn{5}{c}{ Correlação } \\
\cline { 2 - 6 } & V4 & V5 & V6 & V7 & V8 \\
\hline Produtividade dos empregados & $-0,110060$ & 0,131323 & $-0,080813$ & 0,336484 & 0,340185 \\
Satisfação dos empregados & $-0,253045$ & 0,180300 & 0,044779 & 0,255463 & $\mathbf{0 , 6 8 4 1 5 8}$ \\
Rotatividade de empregados & $-0,267745$ & $-0,004150$ & 0,030984 & $-0,382396$ & 0,406234 \\
Flexibilidade dos empregados & $-0,391083$ & $-0,215968$ & $-0,200202$ & $-0,112073$ & $\mathbf{0 , 5 5 8 6 9 0}$ \\
Inovação nos produtos & $-0,279228$ & $-0,138466$ & $-0,055845$ & $-0,041452$ & $\mathbf{0 , 5 6 7 4 6 4}$ \\
Investimentos em treinamento & $-0,256571$ & $-0,247951$ & $-0,228808$ & $-0,080284$ & $\mathbf{0 , 5 1 3 1 4 2}$ \\
Gestão de risco & $-0,366988$ & 0,315068 & 0,267189 & 0,134786 & $\mathbf{0 , 6 7 8 9 2 8}$ \\
\hline Nota. V4 & T Tempo
\end{tabular}

Nota. V4 - Tempo de Atuação da Empresa; V5 - Número de Empregados; V6 - Estrutura Hierárquica; V7 -

Grau de Formalização de Processos Operacionais; e V8 - Prioridade Estratégica.

Analisando os dados disponíveis na Tabela 8, é possível observar que as variáveis V4 (tempo de atuação da empresa), V5 (número de empregados), V6 (estrutura hierárquica) e V7 (grau de formalização de processos operacionais), após a aplicação do coeficiente de correlação de Spearman, apresentaram associações que resultaram enquadradas no nível de magnitude entre negligenciável e fraca, conforme critério adotado para análise da magnitude das correlações.

De acordo com Kaplan e Norton (1997), "os objetivos da perspectiva de aprendizado e crescimento oferecem a infraestrutura que possibilita a consecução de objetivos ambiciosos nas outras três perspectivas" (p. 131), o que não sugerem os resultados da amostra de empresas estudadas, visto que as variáveis supracitadas demonstraram exercer pouca influência na importância que é atribuída aos indicadores da perspectiva aprendizagem e crescimento.

A variável V8 (prioridade estratégica) apresentou, dentro dos parâmetros estabelecidos na pesquisa, correlação positiva, que variou em magnitude de moderada a forte, com quase todos os indicadores de desempenho da perspectiva aprendizagem e crescimento. Esses resultados sugerem que a prioridade estratégica adotada pelas empresas pesquisadas influencia positivamente a importância atribuída aos indicadores de desempenho da perspectiva aprendizagem e crescimento.

Nesse ponto da pesquisa, os resultados encontrados corroboram o estudo realizado por Silva e Callado (2018b), na medida em que ficou evidenciado que as características das empresas impactam na atribuição de importância dispensada aos indicadores de desempenho associados à perspectiva aprendizagem e crescimento do BSC.

Ao final da análise das correlações envolvendo as características das empresas (fatores contingenciais) e os indicadores de desempenho classificados nas perspectivas do 
BSC, restou evidenciado que 23 se mostraram estatisticamente significativas, conforme pode ser observado na Tabela 11.

Tabela 11

Resumo das constatações contidas nas correlações estatisticamente significativas

\begin{tabular}{|c|c|c|c|c|c|}
\hline \multirow{2}{*}{$\begin{array}{c}\text { Perspectivas do } \\
\text { BSC }\end{array}$} & \multicolumn{5}{|c|}{ Características das Empresas x Indicadores de Desempenho } \\
\hline & V4 & V5 & V6 & V7 & V8 \\
\hline Financeira & - & - & $\begin{array}{l}\text { Custos } \\
\text { totais; }\end{array}$ & $\begin{array}{l}\text { Crescimento } \\
\text { das vendas; }\end{array}$ & $\begin{array}{l}\text { Retorno do investi- } \\
\text { mento; Inventários; } \\
\text { Preço dos produtos. }\end{array}$ \\
\hline Clientes & $\begin{array}{l}\text { (Crescimento } \\
\text { da partici- } \\
\text { pação de } \\
\text { mercado) }\end{array}$ & $\begin{array}{l}\text { (Devolução de } \\
\text { produtos); (Res- } \\
\text { ponsividade) }\end{array}$ & $\begin{array}{l}\text { (Devolução } \\
\text { de produ- } \\
\text { tos); }\end{array}$ & - & $\begin{array}{l}\text { Qualidade dos produ- } \\
\text { tos; Tempo de entre- } \\
\text { ga; Pontualidade de } \\
\text { entrega; Crescimento } \\
\text { da participação de } \\
\text { mercado. }\end{array}$ \\
\hline $\begin{array}{l}\text { Processos In- } \\
\text { ternos }\end{array}$ & - & - & - & - & $\begin{array}{l}\text { Tempo de realização } \\
\text { das operações; Flexi- } \\
\text { bilidade de volume } \\
\text { operacional; Uso da } \\
\text { capacidade instalada; } \\
\text { Entregas no prazo. }\end{array}$ \\
\hline $\begin{array}{l}\text { Aprendizagem } \\
\text { e Crescimento }\end{array}$ & - & - & - & $\cdot$ & $\begin{array}{l}\text { Satisfação dos } \\
\text { empregados; Rotati- } \\
\text { vidade de emprega- } \\
\text { dos; Flexibilidade dos } \\
\text { empregados; Inovação } \\
\text { nos produtos; Investi- } \\
\text { mentos em treinamen- } \\
\text { to; Gestão de risco. }\end{array}$ \\
\hline
\end{tabular}

Nota. V4 - Tempo de Atuação da Empresa; V5 - Número de Empregados; V6 - Estrutura Hierárquica; V7 Grau de Formalização de Processos Operacionais; e V8 - Prioridade Estratégica. Negrito - Correlação forte; (Parênteses) - Correlação Inversa.

Observe-se que nas correlações envolvendo as características das empresas e os indicadores de desempenho foram estabelecidas cinco associações de magnitude forte e em todas estavam presentes a variável prioridade estratégica. Essa variável também se mostrou a mais relacionável, estabelecendo correlações estatisticamente significativas com indicadores de desempenho de todas as perspectivas do BSC.

Resultado nesse sentido foi encontrado por Cândido et al. (2016), quando mapeavam as principais características presentes em modelos de sistemas de medição de desempenho da construção civil. Esses autores identificaram que em $59 \%$ dos modelos havia vinculação explícita entre os indicadores e a estratégia. Tais resultados podem ser explicados pelo fato de as empresas necessitarem utilizar sistemas de medição de desempenho que motivem a implementação da estratégia com sucesso (Kaplan \& Norton, 1997). 


\section{CONCLUSÃO}

A pesquisa que originou este artigo foi produzida com o objetivo de investigar o nível das relações estabelecidas entre fatores contingenciais de empresas pertencentes ao setor de construção civil da Cidade de Recife, PE, e a importância atribuída pelos gestores aos indicadores de desempenho classificados nas perspectivas do Balanced Scorecard (BSC). Para atingir o objetivo utilizou-se uma adaptação do questionário contido no protocolo de pesquisa desenvolvido e aplicado por Silva e Callado (2018b). Aos dados coletados foi aplicado o teste não paramétrico coeficiente de correlação de Spearman. Os resultados obtidos com a aplicação do supracitado teste associado ao critério de interpretação da magnitude da correlação proposto por Franzblau (1958 apud Pereira et al., 2016) demonstraram a presença de algumas relações estatisticamente significativas.

No que concerne às relações entre a importância atribuída aos indicadores de desempenho e as características dos gestores, o resultado da aplicação do coeficiente de correlação de Spearman revelou associações estatisticamente significativas apenas nas dimensões clientes e processos internos do BSC. Destaque-se que nas correlações envolvendo as características dos gestores e os indicadores de desempenho só foi estabelecida uma associação de magnitude forte entre a experiência profissional e o indicador desperdícios.

Embora pesquisas apontem que as características pessoais do tomador de decisões influenciem diretamente o diagnóstico, as escolhas e a direção estratégica a ser tomada pela organização (Bressan \& Toledo, 2013), essa característica foi pouco visualizada na pesquisa ora desenvolvida, visto que os atributos do perfil individual dos gestores mostraram influenciar a importância a tribuída ao uso de poucos indicadores de desempenho classificados nas perspectivas do BSC.

Resultados correlatos foram encontrados por Montenegro e Callado (2019), quando investigaram as relações entre fatores contingenciais $e$ indicadores de desempenho na indústria de confecções do vestuário, e por Silva e Callado (2018b), ao pesquisarem os fatores contingenciais e os indicadores de desempenho na construção.

Com relação aos resultados da análise das relações entre a importância atribuída aos indicadores de desempenho e as características das empresas, estes sugerem que as características das empresas pesquisadas podem influenciar as escolhas estratégicas relacionadas aos indicadores de desempenho. Observe-se que as características das empresas compreendem os fatores contingenciais ambiente, porte, estrutura, tecnologia e estratégia.

Nesse contexto, os resultados demonstraram que a variável prioridade estratégica se mostrou a mais relacionável com os indicadores de desempenho em todas as dimensões do BSC, embora as demais variáveis também tenham se mostrado relacionáveis com alguns indicadores de desempenho. As correlações classificadas na magnitude forte entre a variável 
prioridade estratégica ocorreram com os indicadores pontualidade de entrega, flexibilidade de volume operacional, entregas no prazo, satisfação dos empregados e gestão de risco.

Esse resultado pode ser explicado em razão de ter sido adotado nesta pesquisa o modelo de estratégia proposto por Porter (1999), de menor custo e diferenciação, aliado ao fato de que o objetivo dos sistemas de mensuração é motivar a implementação da estratégia organizacional (Kaplan \& Norton, 1997), em que os indicadores são utilizados para monitorar o alcance desse objetivo.

Diante dos resultados apresentados pelos testes de correlação de Spearman, bem como com as análises realizadas nos dados coletados nas empresas da amostra da pesquisa é possível concluir que a importância atribuída aos indicadores de desempenho associáveis às perspectivas do BSC podem ser influenciadas por fatores contingenciais das organizações, resultado esse que corrobora os achados de Montenegro e Callado (2019), que pesquisaram as relações entre fatores contingenciais e indicadores de desempenho na indústria de confecções do vestuário, e Silva e Callado (2018b), que investigaram fatores contingenciais e indicadores de desempenho na indústria da construção civil de João Pessoa, PB.

Quanto às possíveis contribuições desta pesquisa, entende-se que ao estudar a importância dos indicadores de desempenho para as organizações pesquisadas, explorando a existência dos fatores contingenciais como elemento catalisador da configuração organizacional, é provável que o estudo contribua para estimular outros pesquisadores que pretendam desenvolver estudos correlatos em outras localidades e até mesmo em outros segmentos de mercado, bem como pode ser utilizado como parâmetro para reflexão das empresas do setor pesquisado acerca do tema aqui discutido.

Como limitação ao desenvolvimento desta pesquisa tem-se a dificuldade de acesso à amostra, logo se trata de uma amostra obtida por conveniência. $O$ tamanho da amostra também é uma limitação, visto que, pelo fato de se trabalhar com uma amostra reduzida, torna-se inviável fazer inferências, ficando os resultados restritos ao grupo de empresas pesquisadas. Como sugestões para futuras pesquisas sugerem-se estudos mais aprofundados e em outras regiões com o fito de gerar informações mais detalhadas sobre a natureza das relações encontradas nesta pesquisa.

\section{REFERÊNCIAS}

Abdel-Kader, M., \& Luther, R. (2008). The impact of firm characteristics on management accounting practices: A UK-based empirical analysis. The British Accounting Review, 40(1), 2-27. https://doi.org/10.1016/j.bar.2007.11.003 
Araújo, J. G. R., Callado, A. L. C., \& Cavalcanti, B. S. B. (2014). Habilidades e competências do controller: Um estudo com alunos de cursos de pós-graduação em controladoria. Revista Catarinense da Ciência Contábil, 13(38), 52-64. https://doi.org/10.16930/2237-7662/ rccc.v13n38p52-64

Beuren, I. M., \& Fiorentin, M. (2014). Influência de fatores contingenciais nos atributos do sistema de contabilidade gerencial: Um estudo em empresas têxteis do estado do Rio Grande do Sul. Revista de Ciências da Administração, 16(38), 195-212. https://doi. org/10.5007/2175-8077.2014v16n38p195

Bressan, F., \& Toledo, G. L. (2013). A influência das características pessoais do empreendedor nas escolhas estratégicas e no processo de tomada de decisão. Revista Psicologia: Organizações \& Trabalho, 13(3), 309-324. http://pepsic.bvsalud.org/pdf/rpot/v13n3/ vl3n3a08.pdf

Callado, A. L. C., Callado, A. A. C., Holanda, F. M. A., \& Leitão, C. R. S. (2008). Utilização de indicadores de desempenho no setor hoteleiro de João Pessoa - PB. Revista Turismo Visão e Ação, 10(1), 23-38. https://doi.org/10.14210/rtva.v1Onl.p23\%20-\%2038

Cândido, L. F., Lima, S. H. O., \& Barros, J. P., Neto. (2016). Análise de sistemas de medição de desempenho na indústria da construção civil. Ambiente Construído, 16(2), 189-208. https://doi.org/10.1590/s1678-86212016000200087

Cervo, A. L., Bervian, P. A., \& Silva, R. (2007). Metodologia científica (6a ed.). Pearson Prentice Hall.

Chenhall, R. H. (2003). Management control systems design within its organizational context: Findings from contingency-based research and directions for the future. Accounting, Organizations and Society, 28(2-3), 127-168. https://doi.org/10.1016/S0361-3682(01)000277

Chenhall, R. H. (2006). Theorizing contingencies in management control systems research. Handbook of Management Accounting Research, 1, 163-205. https://doi.org/10.1016/S17513243(06)01006-6

Cosso, E. \& Oliveira, M. A. (2013). As teorias organizacionais, os estudos recentes e as possíveis influências na prática das organizações. Revista de Ciências Ambientais e Saúde, 4O(1), 3-12. http://dx.doi.org/10.18224/est.v4Oi1.2686

Donaldson, L. (2015). Structural contingency theory. International Encyclopedia of the Social \& Behavioral Sciences (2nd ed), 23, 609-614. Elsevier. https://doi.org/10.1016/B978-008-097086-8.73110-2 
Espejo, M. M. S. B. (2008). Perfil dos atributos do sistema orçamentário sob a perspectiva contingencial: Uma abordagem multivariada (Tese de Doutorado, Universidade de São Paulo, São Paulo, SP, Brasil). http://www.teses.usp.br/teses/disponiveis/12/12136/tde30062008-141909/

Fleury, A. (1990). Capacitação tecnológica e processo de trabalho: Comparação entre o modelo japonês e o brasileiro. RAE - Revista de Administração de Empresas, 30(4), 23 30. https://doi.org/10.1590/S0034-75901990000400003

Heinzen, D. A. M., Dias, A. B. B. B., \& Marinho, S. V. (2013). Avaliação do sistema de medição de desempenho organizacional de uma instituição de ensino superior de Santa Catarina à luz dos atributos desejáveis. Revista Meta: Avaliação, 5(15), 327-352. http:// dx.doi.org/10.22347/2175-2753v5i15.211

Hyvonen, J. (2007). Strategy, performance measurement techniques and information technology of the firm and their links to organizational performance. Management Accounting Research, 18(3), 343-366. https://doi.org/10.1016/j.mar.2007.02.001

Jacomossi, F. A., Zonatto, V. C. S., \& Lavarda, C. E F. (2018). Inter-relação entre fatores contingenciais e a adoção de sistemas de controle gerencial em empresa de serviço contábil. Anais do Congresso de Contabilidade da UFRGS, Porto Alegre, RS, Brasil, 3. https://www.ufrgs.br/congressocont/index.php/IIIContUFRGS/IIIContUFRGS/paper/ view/126/84

Kaplan, R. S., \& Norton, D. P. (1997). A estratégia em ação: Balanced scorecard. Elsevier.

Klein, L., \& Almeida, L. B. (2017). A influência dos fatores contingenciais na adoção de práticas de contabilidade gerencial nas indústrias paranaenses. Revista Universo Contábil, 13(3), 90-119. https://doi.org/10.4270/RUC.2017320

Leite, E. G., Diehl, C. A., \& Manvailer, R. H M. (2015). Práticas de controladoria, desempenho e fatores contingenciais: Um estudo em empresas atuantes no Brasil. Revista Universo Contábil, 11(2), 85-107. https://doi.org/10.4270/RUC.2015213

Leone, N. M. C. P. G. (1991). A dimensão física das pequenas e médias empresas (P.M.E's): À procura de um critério homogeneizador. RAE - Revista de Administração de Empresas, 31(2), 53-59. https://doi.org/10.1590/S0034-75901991000200005

Maguire, E. R. (2003). Organizational structure in american police agencies: Context, complexity, and control. State University of New York Press. 
Mainardes, E. W., Ferreira, J., \& Raposo, M. (2011). Conceitos de estratégia e gestão estratégica: Qual é o nível de conhecimento adquirido pelos estudantes de gestão. Revista FACEF Pesquisa - Desenvolvimento e Gestão, 14(3), 278-297. http://periodicos.unifacef. com.br/index.php/facefpesquisa/article/view/296

Maltempi, M. M. (2014). Sistemas de medição desempenho organizacional: uma análise comparativa das ferramentas PRISM, PYRAMID e BSC. Revista Universitas, (13), 107 120. http://revistauniversitas.inf.br/index.php/UNIVERSITAS/article/view/173

Marques, K. C. M., \& Souza, R. P. (2010). Pontos críticos da abordagem da contingência nos estudos da Contabilidade gerencial. Anais do Congresso Brasileiro de Custos, Belo Horizonte, MG, Brasil, 17. https://anaiscbc.emnuvens.com.br/anais/article/view/822

Marquezan, L. H. F., Diehl, C. A., \& Alberton, J. R. (2013). Indicadores não financeiros de Avaliação de desempenho: Análise de conteúdo em relatórios anuais digitais. Revista Contabilidade, Gestão e Governança, 16(2), 46-61. https://www.revistacgg.org/contabil/ article/view/533

Montenegro, F. R. M. S., \& Callado, A. L. C. (2019). Fatores contingenciais e o uso de indicadores de desempenho associados às perspectivas do Balanced Scorecard. Revista Gestão Organizacional, 12(1), 73-91. http://dx.doi.org/10.22277/rgo.vl4il.4540

Moreira, E. (2002). Proposta de uma sistemática para o alinhamento das ações operacionais aos objetivos estratégicos, em uma gestão orientada por indicadores de desempenho (Tese de Doutorado, Universidade Federal de Santa Catarina, Florianópolis, SC, Brasil). http://repositorio.ufsc.br/xmlui/handle/123456789/83052

Nakamura, W. T., \& Mineta, R. K. N. (2001). Identificação dos fatores que induzem ao uso do Balanced Scorecard como instrumento de gestão estratégica. Anais do Encontro da Associação Nacional dos Programas de Pós-Graduação em Administração, Campinas, SP, Brasil, 25. http://www.anpad.org.br/admin/pdf/enanpad200l-eso-789.pdf

Oliveira, D. P. R. (2005). Planejamento estratégico: Conceitos, metodologia e práticas (22a ed.). São Paulo: Atlas.

Otley, D. (2016). The contingency theory of management accounting and control: 1980-2014. Management Accounting Research, 31, 45-62. https://doi.org/10.1016/j. mar.2016.02.001

Pereira, J. R., Rezende, L. B. O., Andalécio, A. M. L., Sousa, C. V., \& Matos, E. B. (2016). A gestão do conhecimento em uma instituição de ensino privada. Revista Perspectivas em Gestão \& Conhecimento, 6(2), 113-133. https://dialnet.unirioja.es/servlet/articulo?codigo $=5763761$ 
Porter, M. E. (1999). Competição = on competition: Estratégias competitivas essenciais (12a ed.). Rio de Janeiro: Campus.

Prodanov, C. C., \& Freitas, E. C. (2013). Metodologia do Trabalho Científico [recurso eletrônico]: Métodos e técnicas da pesquisa e do trabalho acadêmico ( $2 a$ ed.). Feevale.

Rossetti, A., \& Morales, A. B. (2007). O papel da tecnologia da informação na gestão do conhecimento. Revista Ciência da Informação, 36(1), 124-135. https://doi.org/10.1590/ S0100-19652007000100009

Santos, D. F. L. (2015). Modelo de gestão financeira aplicada em empresa do setor de construção civil. TAC - Tecnologias de Administração e Contabilidade, 5(2), 119-135. http:// www.anpad.org.br/periodicos/arq_pdf/a_1653.pdf

Santos, F. O., Jesus, S. R. P., Souza, W. A. R., \& Cavalcante, T. S. B. (2016). Avaliação do sistema de controle gerencial sob a influência de fatores contingenciais: Estudo de caso em um grupo econômico. Revista de Contabilidade e Controladoria, 8(2), 23-48. https://revistas.ufpr.br/rcc/article/view/38382

Silva, A. R., \& Callado, A. L. C. (2018a). Aderência de indicadores de desempenho associados às perspectivas do Balanced Scorecard no setor de construção civil do município de João Pessoa (PB). Reunir - Revista de Administração, Ciências Contábeis e Sustentabilidade, 8(2), 23-34. https://doi.org/10.18696/reunir.v8i2.626

Silva, A. R., \& Callado, A. L. C. (2018b). Relações entre fatores contingenciais de empresas do setor da construção civil e a importância atribuída a indicadores de desempenho. Revista Capital Científico - Eletrônica, 16(1), 112-129. https://revistas.unicentro.br/index.php/ capitalcientifico/article/view/5011/pdf

Silva, A. R., Callado, A. A. C., \& Callado, A. L. C. (2016). Análise das relações entre o uso de indicadores de desempenho e fatores contingenciais de empresas de empresas do setor da construção civil. Anais do Congresso Brasileiro de Custos, Porto de Galinhas, PE, Brasil, 23. https://anaiscbc.emnuvens.com.br/anais/article/view/4161/4162

Silva, R. C. E. O., Koyashiki, M. L. M., Cruz, J. A. W., Ahlfeldt, R., Silva, W. V., \& Corso, J. M. D. (2015). Análise de desempenho das ações das empresas do setor da construção civil na Bovespa em relação à rentabilidade, estrutura de capital e conjuntura setorial. Revista Catarinense de Ciência Contábil, 14(41), 9-19. http://dx.doi.org/10.16930/2237-7662/rccc. v14n4lp9-19

Siqueira, K. P. S. (2014). Uma investigação acerca do uso de indicadores de desempenho em empreendimentos hoteleiros situados na Região Metropolitana de Recife (Dissertação de Mestrado, Universidade Federal de Pernambuco, Recife, PE, Brasil). https://repositorio.ufpe.br/handle/123456789/11513 
Sousa, D. S. V., Cândido, L. F., \& Barros, J. P., Neto. (2018). Medição de desempenho na construção civil: Um estudo exploratório com construtoras cearenses. Ambiente Construído, 8(1), 9-29. http://dx.doi.org/10.1590/s1678-86212018000100207

Tezza, R., Bornia, A. C., \& Vey, I. H. (2010). Sistemas de medição de desempenho: Uma revisão e classificação da literatura. Revista Gestão \& Produção, 17(1), 75-93. https://doi. org/10.1590/S0104-530X2010000100007

Tinoco, J. E. P., Assêncio, E. W., João, B. N., \& Claro, J. A. C. S. (2011). Influência dos valores individuais no desempenho empresarial: Um estudo usando o inventário de valores de Schwartz. Revista Sociedade, Contabilidade e Gestão, 6(2), 139-155. https://doi. org/10.21446/scg_ufrj.v6i2.13243

Wernke, R., \& Junges, I. (2017). Níveis de utilização e importância atribuídos aos indicadores não financeiros por empresas da região sul de Santa Catarina. Revista Contemporânea de Contabilidade, 14(03), 55-87. https://doi.org/10.5007/21758069.2017v14n33p55 
